\title{
EVOLUCIÓN DE LA BRECHA SALARIAL POR GÉNERO EN COLOMBIA 2004-2012. UN ANÁLISIS DEL FENÓMENO TECHO DE CRISTAL ${ }^{*}$
}

\author{
Recibido: 07 de diciembre de 2017 - Aprobado: 08 de agosto de 2018 \\ https://doi.org/10.22395/seec.v21n48a1 \\ Camilo Fabiam Gómez Segura** \\ Óscar Hernán Cerquera Losada** \\ Cristian Felipe Orjuela Yacue ${ }^{* * *}$
}

\section{RESUMEN}

El presente trabajo analiza los determinantes de la brecha salarial por género en Colombia durante el periodo 2004-2012, utilizando las encuestas Mesep-DANE de los años 2004, 2008 y 2012. Se realizó la descomposición de la brecha salarial en los diferentes puntos de la distribución incondicional de los salarios. Los resultados obtenidos muestran que existe un diferencial salarial promedio en favor de los hombres y este es creciente en el tiempo, el cual se explica en el $20 \%$ y en el $40 \%$ por factores diferentes a la productividad de los individuos. Al analizar el diferencial salarial por género en los distintos cuantiles de la distribución del ingreso, se encuentra que el efecto característica explica la brecha bruta a medida en que se avanza en la distribución de salarios, este resultado evidencia la existencia de un techo de cristal para las mujeres.

\section{PALABRAS CLAVE}

Discriminación salarial; efecto características; efecto remuneración; techo de cristal.

\section{CLASIFICACIÓN JEL}

J15, J16, J31, J70.

\section{CONTENIDO}

Introducción; 1. Revisión de la literatura; 2. Metodología; 3. Datos y estadísticas descriptivas; 4. Resultados; 5 . Conclusiones; Bibliografía; Anexos.

\footnotetext{
Artículo de investigación. Grupo de investigación Iguaque, categoría C. Nombre del proyecto de investigación Diferencial salarial por género en Colombia. Entidad financiadora, Universidad Surcolombiana. Proyecto ejecutado en el año 2016

* Economista, Universidad Surcolombiana, Neiva, Colombia. Magíster en Economía, Universidad Nacional de La Plata, La Plata, Argentina. Docente catedrático, Facultad de Economía y Administración, Universidad Surcolombiana, Neiva, Huila. Dirección: Av. Pastrana Borrero con Carrera 1 A.A. Neiva, Huila, Colombia. Integrante del Grupo de investigación Iguaque, categoría C. Código Postal 410001. Teléfono: 3214300622. Correo electrónico: camilo.gomez@usco.edu.co.

*** Economista, Universidad Surcolombiana, Neiva, Colombia. Magíster en Economía, Universidad de Buenos Aires, Buenos Aires, Argentina. Docente de planta, Facultad de Economía y Administración, Universidad Surcolombiana, Neiva, Huila. Dirección: Av. Pastrana Borrero con Carrera 1 A.A. Neiva, Huila, Colombia. Integrante del Grupo de investigación Iguaque, categoría C. Código Postal 410001. Teléfono: 3219045533 Correo electrónico: oscar.cerquera@usco.edu.co.

...* Economista, Universidad Surcolombiana, Neiva, Colombia. Magíster en Economía, Universidad Nacional de La Plata, La Plata, Argentina. Docente de tiempo completo en posgrados, Universidad Uniminuto, Neiva, Huila. Dirección: Calle 15 N. ${ }^{0}$ 5 -58, Neiva, Huila, Colombia. Integrante del Grupo de investigación Iguaque, categoría C. Código Postal 410001. Teléfono: 3195290053. Correo electrónico: cristian.orjuela10@gmail.com.
} 


\title{
EVOLUTION OF THE SALARIAL GAP BY GENDER IN COLOMBIA 2004-2012. AN ANALYSIS OF THE GLASS CEILING PHENOMENON
}

\begin{abstract}
This paper analyzes the determinants of the wage gap by gender in Colombia from 2004 to 2012, using the Mesep-DANE surveys of 2004, 2008 and 2012. A breakdown of the wage gap in the different points of the unconditional distribution of wages was carried out. The results obtained show that there is an average wage differential in favor of men and that it is growing over time. It is explained in $20 \%$ and $40 \%$ by factors other than the productivity of individuals. When analyzing the wage difference by gender in the different quantiles of the income distribution, it was found that the characteristic effect explains the gross gap as one advances through the wage distribution. This result demonstrates the existence of a glass ceiling for women.
\end{abstract}

\section{KEYWORDS}

Salary discrimination; characteristic effect; remuneration effect; glass ceiling.

\section{JEL CLASSIFICATION}

J15, J16, J31, J70.

\section{CONTENT}

Introduction; 1. Literature review; 2. Methodology; 3. Data and descriptive statistics; 4. Results; 5. Conclusions; Bibliography; Annexes.

\section{AVALIAÇÃO DA BRECHA SALARIAL POR GÊNERO NA COLÔMBIA 2004-2012. UMA ANÁLISE DO FENÔMENO “TETO DE VIDRO”}

\section{RESUMO}

Este trabalho analisa os determinantes da brecha salarial por gênero na Colômbia entre 2004 e 2012 utilizando as pesquisas da Missão para a União das Séries de Emprego, Pobreza e DesigualdadeDepartamento Administrativo Nacional de Estatística, dos anos 2004, 2008 e 2012. Realizou-se a análise da brecha salarial nos diferentes pontos da distribuição incondicional dos salários. Os resultados obtidos mostram que existe um diferencial salarial médio em favor dos homens, o qual cresce no tempo, o que se explica em $20 \%$ e em $40 \%$ por fatores diferentes da produtividade dos indivíduos. Ao analisar o diferencial salarial por gênero nas diferentes partes da distribuição do ingresso, verifica-se que o efeito característica explica a brecha bruta à medida que se avança na distribuição de salários; esse resultado evidencia a existência de um "teto de vidro" (glass ceiling) para as mulheres.

\section{PALAVRAS-CHAVE}

Discriminação salarial; efeito características; efeito remuneração; "teto de vidro" (glass ceiling).

\section{CLASSIFICAÇÃO JEL}

J15, J16, J31, J70.

\section{CONTEÚDO}

Introdução; 1. Revisão da literatura; 2. Metodologia; 3. Dados e estatísticas descritivas; 4. Resultados;

5. Conclusões; Bibliografia; Anexos. 


\section{INTRODUCCIÓN}

El ingreso laboral es uno de los componentes más importantes en el mercado laboral, debido a que es una variable que influye de manera directa en el bienestar individual. Sin embargo, este depende de ciertas características tanto del individuo como del propio mercado, lo cual hace que exista heterogeneidad en el salario. El problema surge cuando esta dispersión esta explicada por características observables que en cierto modo no infieren productividad, como es el caso del género o la raza.

En Colombia, el diferencial salarial por género ha decrecido en las últimas décadas debido al incremento en la participación laboral que, a su vez, se puede explicar por los grandes cambios y transformaciones sociales y culturales que han permitido a la mujer, alcanzar mayor acceso al sistema educativo, incrementar su probabilidad de empleo, aumentar su experiencia laboral, en otras palabras, las mujeres pasaron a complementar el mercado de trabajo (Flórez, 2004).

En los últimos 50 años, la participación laboral de las mujeres en Colombia ha estado en aumento al pasar de $19 \%$ a $39 \%$, en el periodo comprendido entre 1950 y 1985 y, de manera simultánea, se ha presentado una mejora en su educación, como también una especie de movilidad hacia actividades económicas con mayores ingresos (Tenjo, 1993). Este resultado se asemeja a los obtenidos por Urdinola (1998), el cual reporta que en Colombia, durante el periodo 1982-1997, para las siete principales ciudades del país, el género femenino tuvo un incremento en la participación laboral del $36 \%$ al $48 \%$ respectivamente.

Así mismo, el incremento de la participación de la mujer en el mercado laboral ha traído consigo una disminución de la desigualdad en los ingresos laborales, tanto así que para 1976 era del 36 \% y para 1995 ese diferencial salarial se redujo al 21 \% (Ribero y Meza, 1997). Este hecho ha incentivado la realización de trabajos que analizan el efecto de la creciente participación de la mujer en el mercado laboral, ya que con el paso del tiempo las mujeres han cambiado su tradicional rol en la sociedad -como amas de casa-, esta situación conlleva a un incremento en sus aportes en el hogarl.

Un ejemplo de las diversas investigaciones generadas por este fenómeno, son los trabajos que estudian el diferencial salarial por género, como los de Becker (1971), Arrow (1971), Oaxaca (1973), Blinder (1973), Machado y Mata (2004), Melly (2005), Autor, Katz y Kearney (2005) y Firpo, Fortin y Lemieux (2011). Trabajos que han contribuido a analizar este fenómeno económico a través de roles de género, estructura salarial

Entiéndase como aportes al hogar todas las actividades diarias no remuneradas que la mujer desempeña para mantener su familia u hogar. 
y, especialmente, de la discriminación laboral, que se ha convertido en una valiosa alternativa para analizar la problemática. No obstante, tiene la limitación de solo considerar los determinantes de la brecha en el promedio de los salarios, en otras palabras, no se estudia qué sucede en toda la distribución del ingreso laboral. Así mismo, no existen trabajos en Colombia que planteen una metodología que permita controlar el sesgo de selección en cada punto de la distribución del ingreso.

Es por ello que este trabajo es pionero, no solamente porque busca contribuir al estudio del diferencial salarial por género en Colombia al estudiar la evolución en los últimos años de la brecha salarial para los ingresos promedios, sino que emplea una metodología que permite controlar el sesgo de selección para toda la distribución del ingreso laboral, lo cual permite analizar mejor si el diferencial salarial se incrementa a medida que se avanza en la distribución de salarios, es decir, determinar si existe evidencia del fenómeno de techo de cristal.

Se entiende por techo de cristal el conjunto de barreras que limitan el ascenso de las mujeres a mejores cargos y, por ende, a mayores remuneraciones en el mercado laboral. Estas barreras se explican por los diferentes obstáculos (esto incluye la discriminación salarial por género), los cuales generan un techo profesional que solamente les permite establecerse en puestos de trabajos que las ubica en la parte intermedia en la distribución de salarios, a pesar de tener un capital humano suficiente para obtener puestos de trabajo con mejores ingresos.

Esta investigación cuenta con información para el periodo 2004-2012, tiempo en que se crea la Ley 1496 de 2011, cuyo objetivo es el de garantizar la igualdad salarial por género, al fijar los mecanismos necesarios para que dicha igualdad sea real. Para ello, se crean diez artículos que buscan ajustar la igualdad de trabajadores y trabajadoras (artículo 2), definir los conceptos de discriminación directa e indirecta (artículo 3), crear los factores de valoración salarial (artículo 4), realizar un registro de perfil y asignación de cargos por sexo por parte de los empresarios (artículo 5), instaurar auditorías a las empresas que permitan verificar la igualdad salarial (artículo 6), estructurar las condiciones de trabajo y salario (artículo 7), promover y fortalecer el acceso de las mujeres al mercado laboral urbano y rural (artículo 8) y, finalmente, establecer el seguimiento a través de nuevas variables que se incorporan en la Gran encuesta integrada de hogares (GEIH) (artículo 9).

Para poder analizar el comportamiento del diferencial salarial se utilizan las encuestas empalmadas Mesep-DANE² para los años 2004, 2008 y 2012, este periodo es relevante debido a que se evalúa la brecha salarial por género junto a sus deter-

Misión para el Empalme de las Series de Empleo, Pobreza y Desigualdad (Mesep) y Departamento Administrativo Nacional de Estadística (DANE). 
minantes, antes y después de aplicar la primera ley que busca garantizar la igualdad salarial entre hombres y mujeres. La metodología empleada es propuesta por Melly (2005), la cual plantea descomposiciones de la brecha salarial en diferentes puntos de la distribución incondicional de los salarios.

El resto del documento se organiza de la siguiente manera: en la sección 1 se realiza una revisión de la literatura teórica y empírica, como también antecedentes en América Latina y Colombia; la sección 2 describe la metodología utilizada para la estimación y descomposición del diferencial salarial; la sección 3 presenta los datos con las variables empleadas y las estadísticas descriptivas; en la sección 4 se muestran y analizan los resultados obtenidos y, por último, en la sección 5 se muestran las conclusiones.

\section{REVISIÓN DE LA LITERATURA}

Fernández (2006) afirma que "no toda diferencia de salarios es discriminación, ni toda discriminación se traduce en diferencias en salarios" (p.5). Existe discriminación salarial cuando dos individuos con iguales características productivas (educación, edad, experiencia, laboral, etc.) y habilidades, realizan el mismo trabajo, pero uno de ellos obtiene mayor remuneración que el otro. De igual manera se pueden establecer otras discriminaciones laborales en cuanto a la probabilidad de acceder a un trabajo ${ }^{3}$, al tratamiento diferente en los beneficios laborales y a las oportunidades de ascenso.

\subsection{Evidencia teórica}

La literatura internacional plantea ciertos enfoques que pueden explicar, bajo algunos supuestos, los diferenciales salariales por género. Uno de los más importantes es la teoría económica de la discriminación ${ }^{4}$, que se divide en la teoría de discriminación por gustos o preferencias desarrollada por Becker (1971) y la teoría de discriminación estadística desarrollada por Arrow (1971) y Phelps (1972).

La teoría de la discriminación por gustos o preferencias, desarrollada por Becker, se basa en el prejuicio de los agentes en el mercado laboral (empleador, empleado y consumidor). La idea consiste en que los agentes incurren en costos o están dispuestos a pagar para evitar ciertos grupos (minorías). Según Becker la discriminación está representada por un coeficiente $\delta$ también conocido como coeficiente de desagrado, este coeficiente se relaciona

3 Lo cual establece que a pesar que dos individuos tengan iguales características productivas, uno de ellos tenga mayor probabilidad de acceder a un empleo.

${ }_{4}$ Este enfoque hace parte del modelo de discriminación competitivo, en el cual los individuos solo buscan maximizar sus beneficios. 
con las características del trabajador que observa el empleador y no con la productividad de los empleados. Entonces, un empleador es discriminador si paga un salario $w$ al grupo discriminado (B) y $w+\delta$ al grupo no discriminado (A), a pesar de que ambos grupos tengan las mismas características productivas.

Por su parte, la teoría de discriminación estadística surge cuando un empleador infiere características productivas del trabajador a partir de rasgos observables (raza y género). Esto se debe a que las empresas tienen información limitada de las habilidades de los individuos, que a su vez es costosa de adquirir. Por ello, el empleador basa sus decisiones en las características observables que no afectan la productividad, pero que pueden estar correlacionadas con características no observables que la afectan. Sin embargo, con el paso del tiempo, el empleador puede constatar la productividad del individuo, lo que conlleva a que la brecha salarial no se explique por las características observables que utilizó el empleador para inferir productividad.

La discriminación estadística surge por inferir productividad en un individuo con base en la pertenencia de este a cierto grupo, y no por tener en cuenta sus características y habilidades individuales. Por ejemplo, como lo plantea Fernández (2006), en un trabajo que requiera cierta fuerza física, una mujer que aspira al trabajo puede no ser seleccionada por el empleador (el cual infiere su productividad para evitar incurrir en costos elevados) debido a la creencia de que las mujeres tienen menor fuerza física con respecto a los hombres.

\subsection{Evidencia empírica}

La literatura empírica internacional y nacional ha tenido una serie de dificultades al tratar de contrastar cuáles factores inciden en el diferencial salarial entre hombres y mujeres, y han asignado mayor peso a los roles de género que a los factores de tipo discriminatorio. Sin embargo, esto no quiere decir que no se hayan desarrollado estudios empíricos que analicen el diferencial salarial por género con la teoría de la discriminación. Ahora bien, las dificultades en la estimación y obtención de información adecuada han sido una de las causas que restringen, y a su vez incentivan, el estudio de esta problemática.

Uno de los trabajos empíricos más importantes fue el realizado por Goldin y Rouse (2000). Ellos buscaron establecer la probabilidad de ingreso de la mujer a las orquestas filarmónicas en Estados Unidos para el periodo de 1970 a 1995, a través de un cuasi experimento en el cual cambia la metodología de ingreso en un esquema de audiciones a ciegas. Los autores encontraron que, bajo este esquema, las mujeres 
tenían un 50 \% más de probabilidad de avanzar a otra ronda en la audición lo que incrementaba, a su vez, la probabilidad de que sea seleccionada finalmente.

En Latinoamérica existen muchos trabajos que tratan esta problemática. Sin embargo, se destaca por completo la investigación desarrollada por Bernat, Ribero y Tenjo (2005) que analiza la situación de la mujer en el mercado laboral para seis países de América Latina (Argentina, Brasil, Colombia, Costa Rica, Honduras y Uruguay) durante las dos últimas décadas del siglo XX. Los autores encuentran que la brecha salarial por género presenta una disminución significativa ${ }^{5}$ debido a la preferencia de los hombres por trabajar más horas a la semana. Así mismo, la segmentación laboral ${ }^{6}$ contribuye al diferencial salarial por género, ya que, si bien las mujeres se concentran en las actividades laborales con mayor salario, sus remuneraciones son menores en los sectores y ocupaciones.

En Colombia se han realizado diversos trabajos que estudian el diferencial salarial por género, sin embargo, la mayoría se han concentrado en analizar este fenómeno a través de la discriminación salarial. Para ello, se utiliza el procedimiento de estimar ecuaciones de salario ${ }^{7}$ y descomponer el diferencial salarial con la metodología de Oaxaca (1973) y Blinder (1973). Uno de los primeros trabajos fue el realizado por Tenjo (1993), el cual estudió la brecha salarial por género en Colombia durante el periodo de 1976 a 1985. Con ayuda de la Encuesta nacional de hogares (ENH), este autor encuentra que, si bien existe una brecha salarial entre hombres y mujeres, esta no solo es explicada por las diferencias en las características observables (educación, edad, etc.), también lo es, por el término de discriminación (efecto remuneración), el cual tuvo un comportamiento creciente. Abadía (2005) estudió la discriminación estadística por sexo en el mercado laboral colombiano para tres grupos de individuos: hijos del jefe del hogar de 30 o menos años, de 20 años o más casados o en uniones libres y solteros de 20 años o más. La idea que proponía Abadía es que, existe discriminación estadística por parte de las firmas, si con el paso de los años, el salario del individuo está cada vez menos relacionado con sus características observables como lo son la edad y la educación ${ }^{8}$.

Por otro lado, un estudio importante fue el desarrollado por Fernández (2006), el cual analizó los determinantes del diferencial por género en Colombia, para el periodo 1997-2003 a través de la Encuesta de calidad de vida (ECV). Este trabajo se

Especialmente en Argentina, Colombia y Honduras.

6 Se entiende que el mercado laboral está segmentado al existir barreras que restringen el ingreso al mercado laboral de trabajadores independiente de su capital humano.

7 También conocidas como ecuaciones de Mincer.

8 Debido a que es una parte del efecto discriminación que se obtiene con las diferentes metodologías de descomposición salarial. 
destaca por ser el primero en utilizar la metodología de regresión por cuantiles (quantile regression) y la descomposición del diferencial salarial realizado por Machado y Mata (2005), al estudiar los determinantes de la brecha salarial en los diferentes puntos de la distribución de salarios. Los resultados obtenidos muestran que existen diferencias salariales entre hombres y mujeres en los valores extremos en la distribución de salarios, como es el caso del percentil 95, en el cual las mujeres ganan un $11,5 \%$ menos con respecto a los hombres para el año 2003. La autora establece que este diferencial no se explica por diferencias en las características productivas entre hombres y mujeres, sino por la remuneración salarial diferente que percibe por género.

Por último, el trabajo realizado por Galvis (2010), utiliza la GEIH durante el año 2009, y estima el diferencial salarial por género en los diferentes puntos de la distribución del ingreso laboral en las trece principales ciudades de Colombia. Los resultados obtenidos sugieren que las brechas salariales no son explicadas por las características observables del individuo, tales brechas se deben, en su mayoría, a la remuneración de los atributos productivos, lo cual implica la posible existencia de discriminación salarial por género. El estudio arroja que las ciudades con mayor concentración de la actividad económica (Bogotá, Medellín, Cali, Manizales y Pereira), tienen menor brecha bruta con respecto a las ciudades periféricas, las cuales tienen mayor discriminación salarial.

\section{METODOLOGÍA}

Como se explicó en la revisión de la literatura, el diferencial salarial entre hombres y mujeres puede deberse a diversos componentes del mercado laboral. Es por ello que la literatura empírica se ha encargado de estudiar el diferencial salarial al responder la siguiente pregunta: ¿qué sucedería con la brecha salarial entre hombres y mujeres si no hubiera diferencias en sus características productivas observables? La metodología de Oaxaca (1973) y Blinder (1973) trata de responder este interrogante descomponiendo la brecha salarial entre hombres y mujeres a través de las funciones de salarios.

$$
\ln \left(w_{j}\right)=\mathrm{X}_{j} \beta_{j}+v_{j} ; \quad j=h, m
$$

Donde $j=h, m$ indica el grupo que pertenece a los hombres ( $h$ ) o las mujeres $(m), \ln \left(w_{j}\right)$ es el logaritmo del salario horario, $X_{j}$ es la matriz de características observables que intervienen en la productividad, $\beta_{j}$ es un vector de coeficientes que presenta los retornos que se obtienen en el mercado de las características observables en $\mathrm{X}_{j}$ para cada grupo $j$. Para finalizar $v_{j}$ es un término no observable que se distribuye con media condicional cero $\left[E\left(v_{j} \mid X\right)=0\right]$ y 
varianza $\sigma^{2}$. Entonces al descomponer el logaritmo del salario horario entre hombres y mujeres se obtiene:

$$
\ln \left(w_{h}\right)-\ln \left(w_{m}\right)=\left(\mathrm{X}_{h} \beta_{h}-\mathrm{X}_{m} \beta_{m}\right)+\left(v_{h}-v_{m}\right)
$$

Puesto que el género es una variable binaria que puede tomar el valor de uno si es hombre $\left(D_{h}=1\right)$ y cero si es mujer $\left(D_{h}=0\right)$, entonces la ecuación de salarios para hombres y mujeres se estima en sus medias muestrales.

$$
\bar{w}_{h}^{\prime}=\overline{\mathrm{X}}_{h} \hat{\beta}_{h} ; \bar{w}_{m}^{\prime}=\overline{\mathrm{X}}_{m} \hat{\beta}_{m}
$$

Donde $\bar{w}_{j}^{\prime}$, es el promedio del salario horario para hombres y mujeres, $\overline{\mathrm{X}}_{j}$ es la media de las características observables y $\hat{\beta}_{j}$ son los coeficientes estimados. Ahora se construye un término contrafactual, el cual muestra el salario que tendrían las mujeres si fueran remuneradas como los hombres $\overline{\mathrm{X}}_{m} \hat{\beta}_{h}$, y se obtiene:

$$
\hat{\Delta}_{w}=\underbrace{\left(\overline{\mathrm{X}}_{h}-\overline{\mathrm{X}}_{\mathrm{i}}\right) \hat{\beta}_{h}}_{\text {Efecto caraterísticas }}+\underbrace{\overline{\mathrm{X}}_{m}\left(\hat{\beta}_{h}-\hat{\beta}_{m}\right)}_{\text {Efecto remuneración }}
$$

El primer componente es la diferencia en el salario promedio de los hombres y el término contrafactual de las mujeres si son remuneradas como los hombres. En otras palabras, es la parte que explica la brecha salarial por las diferencias en las características productivas entre hombres y mujeres y que es conocido como efecto característica. Por su parte, el segundo componente es la diferencia en el ingreso promedio contrafáctico 9 y el salario promedio observado de las mujeres, ya que solo interesa la diferencia de la remuneración en las características observables $^{10}$, este componente se conoce como efecto remuneración y evalúa la posible discriminación salarial.

No obstante, debido a su rol de madre, las mujeres acceden al mercado siempre y cuando su salario sea mayor al salario del mercado, el cual le permita cubrir el costo de oportunidad en el desempeño de labores de madre y ama de casa, lo cual genera un sesgo de selección. Para corregir este sesgo se aplica la metodología de Heckman (1979). Primero se estima la probabilidad de que el salario de los individuos se explique al utilizar como instrumento la tenencia de hijos en el hogar menores de 18 años junto al resto de características observables.

$$
\operatorname{Pr}\left(w_{h, m} \mid \mathrm{X}_{h, m}\right)=\theta\left(\mathrm{X}_{h, m}^{\prime} \delta\right)
$$

\footnotetext{
9 Es el ingreso promedio que recibirán los hombres si tuvieran las características productivas de las mujeres.

10 Debido a que se controlan las diferencias en las características productivas.
} 
Una vez calculada en la primera etapa la probabilidad del salario, junto a las características que influyen en el rol de madre, se estima la inversa de la razón de Mills ${ }^{11}\left(\lambda_{h, m}\right)$, la cual se introduce en la segunda etapa como una variable explicativa adicional que corrige el sesgo de selección.

$$
\hat{\Delta}_{w}-\left(\hat{\gamma}_{h} \lambda_{h}-\hat{\gamma}_{m} \lambda_{m}\right)=\underbrace{\left(\bar{X}_{h}-\bar{X}_{m}\right) \hat{\beta}_{h}}_{\text {Efecto caraterísticas }}+\underbrace{\bar{X}_{m}\left(\hat{\beta}_{h}-\hat{\beta}_{m}\right)}_{\text {Efecto remuneración }}
$$

No obstante, si se quiere analizar la descomposición del diferencial salarial en la distribución incondicional (como los cuantiles) del ingreso laboral, y si se desea saber qué puede pasar con los efectos característica y remuneración, es decir, poder aislar ambos efectos de la distribución no condicional, se utilizan en principio la regresión por cuantiles y las metodologías de descomposición desarrolladas por Machado y Mata (2004), Autor, Katz y Kearney (2005), Melly (2005) y Firpo, Fortin y Lemieux (2011). En este caso, el método de Melly (2005) surge como alternativa para estimar los efectos característica y remuneración en la distribución incondicional del salario, al tener en cuenta el problema de sesgo por selección en el ingreso laboral de las mujeres. Para ello, propone un estimador no condicional que puede generarse al integrar la distribución condicional en todo el rango de la distribución de regresores.

Sin embargo, cuenta con un problema de falta de monotonicidad, es decir, $\theta_{j} \leq \theta_{k}$ no necesariamente implica $\mathrm{X}_{i} \hat{\beta}\left(\theta_{j}\right) \leq \mathrm{X}_{i} \hat{\beta}\left(\theta_{\bar{k}}\right)$. Para evitar esto, propone un cambio de variable en el proceso de integración. Dado esto y a través de Koenker y Bassett (1978) se obtiene:

$$
\mathrm{F}_{\mathrm{Y} \mid \mathrm{X}}^{-1}(\theta \mid \mathrm{X})=\mathrm{X}_{j} \beta(\theta)
$$

Donde $\mathrm{F}_{\mathrm{Y} \mid \mathrm{X}}^{-1}(\theta \mid \mathrm{X})$ es el $\theta$-ésimo cuantil de Ycondicional sobre X. Como se sabe, la relación lineal entre los cuantiles de Y y Xes similar a la relación lineal entre las variables explicativas y explicada bajo Mínimos Cuadrado Ordinarios. Entonces al reemplazar $\mathrm{F}_{\mathrm{Y} \mid \mathrm{X}}^{-1}(\theta \mid \mathrm{X})$ por su estimador consistente $\mathrm{X}_{\mathrm{j}} \beta(\theta)$ y al tomar el ínfimo del conjunto de soluciones finitas sin que sea la única solución se obtiene:

$$
\hat{q}(\hat{\beta}, X)=\inf \left\{q: \frac{1}{N} \sum_{i=1}^{N} \sum_{j=1}^{J}\left(\theta_{j}-\theta_{j-1}\right) 1\left(X_{i} \beta(\theta) \leq q\right) \geq \tau\right\}
$$

"Cociente entre la función de densidad y la función de densidad acumulada de la normal estándar. 
Donde $\hat{q}$ es consistente y distribuido como una normal, por su parte la dificultad de estimar la varianza asintótica conlleva a utilizar el método de Bootstrap para hacer inferencia estadística. La descomposición de los diferenciales en la distribución del salario para hombres y mujeres se realiza a partir de la distribución incondicional. Los estimadores obtenidos se utilizan para poder generar el ingreso contrafáctico en ambos géneros, ya que la idea principal de este estimador es la posibilidad de simular las distribuciones hipotéticas que se puedan utilizar para descomponer las diferencias en la distribución.

Para obtener el efecto en los cambios de las características, remuneración (incluye el efecto residual), se debe primero calcular la distribución contrafactual del salario de las mujeres y suponer que la distribución de sus características observables ha sido como la de los hombres. Entonces, al minimizar (8) para la distribución de los hombres y a través de los coeficientes estimados para las mujeres, se genera:

$$
\hat{q}\left(\beta_{m}, X_{m}\right)-\hat{q}\left(\beta_{h}, X_{h}\right)=\underbrace{\left[\hat{q}\left(\beta_{\text {mediam } r}, X_{m}\right)-\hat{q}\left(\beta_{h}, X_{m}\right)\right]}_{\text {Efecto remuneración }}+\underbrace{\left[\hat{q}\left(\beta_{h}, X_{m}\right)-\hat{q}\left(\beta_{h}, X_{h}\right)\right]}_{\text {Efecto características }}
$$

Para corregir por sesgo de selección en la distribución del salario horario se sigue a Buchinsky (1998), dado esto se utiliza la estimación paramétrica de la inversa de la ratio de Mills y su valor cuadrado, los cuales se agregan como variables adicionales al método de descomposición del diferencial salarial por género propuesto por Melly (2005). Esto permitirá corregir el sesgo de selección debido al rol de madres de las mujeres en cada cuantil de la distribución salarial.

$$
\hat{q}\left(\beta_{m}^{\prime}, X_{m}^{\prime}\right)-\hat{q}\left(\beta_{h}^{\prime}, X_{h}^{\prime}\right)=\underbrace{\left[\hat{q}\left(\beta_{\text {mediam,rr }}^{\prime}, X_{m}^{\prime}\right)-\hat{q}\left(\beta_{h}^{\prime}, X_{m}^{\prime}\right)\right]}_{\text {Efecto remuneración }}+\underbrace{\left[\hat{q}\left(\beta_{h}^{\prime}, X_{m}^{\prime}\right)-\hat{q}\left(\beta_{h}^{\prime}, X_{h}^{\prime}\right)\right]}_{\text {Efecto características }}
$$

\section{DATOS Y ESTADÍSTICAS DESCRIPTIVAS}

Para analizar el diferencial salarial por género en Colombia durante los años 2004 2008 y 2012 se utilizaron las encuestas empalmadas del programa Mesep-DANE, debido a que se especializan en estudiar el mercado laboral e identificar las diferentes variables sociodemográficas que inciden en la vida de los individuos. Así mismo, la Mesep se caracteriza por ser una metodología de empalme entre la Encuesta continua de hogares (ECH) y La GEIH ${ }^{12}$, debido a que el periodo estudiado está sujeto a cambios metodológicos en las encuestas de hogares ${ }^{13}$.

\footnotetext{
12 La Encuesta continua de hogares se desarrolló para el periodo 2000-2005, por su parte la Gran encuesta integrada de hogares empieza a partir del año 2008.

13 La metodología corrige la construcción de variables como ingreso, además corrige la omisión de variables, valores extremos, entre otros.
} 
En este trabajo se utiliza como grupo de estimación a los trabajadores con edad entre 18 y 65 años y con ingresos laborales positivos en su trabajo principal. Así mismo, se construyó la variable de salario por hora, al tener en cuenta los lineamientos en la homogenización de los datos, y la construcción de variables de ingreso. Por otra parte, los trabajadores del servicio doméstico y los jornaleros se tomaron en las estimaciones como un solo tipo de trabajo, dado que son trabajos que se caracterizan por tener poca cualificación y baja remuneración.

La tabla 1 muestra el salario promedio por género y nivel educativo durante el periodo 2004-2012. Como se puede observar, la brecha promedio en el ingreso laboral horario se ha disminuido a favor de las mujeres con el paso del tiempo, al pasar de 23,3 \% en el año 2004 a 17,7 \% durante el 2012. Al estimar los cuantiles 10, 50 y 90 del salario por hora entre hombres y mujeres, se encuentra una reducción del diferencial salarial, a excepción del $10 \%$ de los individuos con menor ingreso, el cual pasó de tener una brecha de 12,6 \% en 2004 a 15,1 \% para el año 2012. Una vez estimados los cuantiles del salario, se obtuvieron los rangos intercuantílicos (RQ) 90-10, 90-50 y 50-10. Se observa que la desigualdad salarial por género se ha reducido en los individuos con menores salarios (rango intercuantílico 50-10), al pasar de 14,8\% en 2004, 7 \% en 2008, a -3 \% para 2012.

Con respecto al salario promedio por género y nivel educativo, se obtuvo que los hombres en promedio remuneran más ingreso laboral por hora con relación a las mujeres en todo nivel educativo. Así mismo, se puede observar que la brecha en el salario hora se incrementa con el tiempo, al pasar en 2004 de 3,7 \%, 28,8 \% y $21,7 \%$ para básica primaria, media y superior respectivamente, a registrar una brecha en 2012 de $8,4 \%, 35,7 \%$ y 30,7 \%.

En cuanto a la desigualdad salarial por género, en cada nivel educativo, las estimaciones muestran que el nivel de educación primaria es el único que presenta una disminución en la desigualdad salarial para todos los rangos intercuantílicos. Por el contrario, los niveles de educación media y superior, presentan una disminución en su diferencial salarial en los individuos con menores ingresos (rango intercuantílico 50-10), los demás rangos muestran un incremento en la desigualdad salarial; sin embargo, son los individuos de mayores salarios (rango intercuantílico 90-50) y educación superior los de mayor crecimiento, al pasar de 14,2 \% en 2004 a $58,3 \%$ para 2012. 
Evolución de la brecha salarial por género en Colombia 2004-2012. Un análisis del fenómeno techo de cristal

Tabla 1. Salario horario promedio por género y nivel educativo.

\begin{tabular}{|c|c|c|c|c|c|c|}
\hline & \multicolumn{2}{|c|}{2004} & \multicolumn{2}{|c|}{2008} & \multicolumn{2}{|c|}{2012} \\
\hline & Mujer & Hombre & Mujer & Hombre & Mujer & Hombre \\
\hline \multicolumn{7}{|c|}{ Total } \\
\hline Salario & $4.168,00$ & $5.435,00$ & $4.269,60$ & $5.498,40$ & $4.025,80$ & $4.892,80$ \\
\hline Quantil 10 & 358,4 & 410,1 & 568,1 & 604,8 & 558,9 & 658 \\
\hline Quantil 50 & $1.602,90$ & $1.870,10$ & $2.169,10$ & $2.325,50$ & $2.171,90$ & $2.224,60$ \\
\hline Quantil 90 & $9.662,20$ & $15.584,30$ & $10.500,00$ & $13.888,80$ & $9.146,50$ & $11.560,20$ \\
\hline $\mathrm{RQ}(90-10)$ & $9.303,80$ & $15.174,20$ & $9.931,90$ & $13.284,00$ & $8.587,60$ & $10.902,20$ \\
\hline $\mathrm{RQ}(90-50)$ & $8.059,30$ & $13.714,20$ & $8.330,90$ & $11.563,30$ & $6.974,60$ & $9.335,60$ \\
\hline $\mathrm{RQ}(50-10)$ & $1.244,50$ & $1.460,00$ & $1.601,00$ & $1.720,70$ & $1.613,00$ & $1.566,60$ \\
\hline \multicolumn{7}{|c|}{ Primaria } \\
\hline Salario & $1.382,00$ & $1.435,40$ & $1.493,20$ & $1.852,00$ & $1.686,80$ & $1.841,70$ \\
\hline Quantil 10 & 257,1 & 216,4 & 390,6 & 425 & 398,1 & 490,3 \\
\hline Quantil 50 & 935 & 900 & $1.181,80$ & $1.250,00$ & $1.341,40$ & $1.419,50$ \\
\hline Quantil 90 & $3.116,80$ & $2.857,10$ & $2.857,10$ & $3.298,60$ & $3.241,90$ & $3.353,70$ \\
\hline $\mathrm{RQ}(90-10)$ & $2.859,70$ & $2.640,70$ & $2.466,50$ & $2.873,60$ & $2.843,80$ & $2.863,40$ \\
\hline $\mathrm{RQ}(90-50)$ & $2.181,80$ & $1.957,10$ & $1.675,30$ & $2.048,60$ & $1.900,50$ & $1.934,20$ \\
\hline $\mathrm{RQ}(50-10)$ & 677,9 & 683,6 & 791,2 & 825 & 943,3 & 929,2 \\
\hline \multicolumn{7}{|c|}{ Media } \\
\hline Salario & $3.174,00$ & $4.454,80$ & $2.463,40$ & $3.999,80$ & $2.487,00$ & $3.692,40$ \\
\hline Quantil 10 & 458,3 & 577,2 & 571,4 & 764,7 & 578,2 & 859,9 \\
\hline Quantil 50 & $1.714,20$ & $2.281,10$ & $1.893,90$ & $2.568,30$ & $1.956,30$ & $2.502,70$ \\
\hline Quantil 90 & $7.064,90$ & $9.023,00$ & $5.000,00$ & $7.246,30$ & $4.471,60$ & $6.956,00$ \\
\hline $\mathrm{RQ}(90-10)$ & $6.606,60$ & $8.445,30$ & $4.428,60$ & $6.481,60$ & $3.893,40$ & $6.095,90$ \\
\hline $\mathrm{RQ}(90-50)$ & $5.350,70$ & $6.741,40$ & $3.106,10$ & $4.678,00$ & $2.515,30$ & $4.453,10$ \\
\hline $\mathrm{RQ}(50-10)$ & $1.255,90$ & $1.703,90$ & $1.322,50$ & $1.803,60$ & $1.378,10$ & $1.642,80$ \\
\hline \multicolumn{7}{|c|}{ Superior } \\
\hline Salario & $11.168,90$ & $14.257,50$ & $8.931,30$ & $12.684,90$ & $8.578,50$ & $12.381,40$ \\
\hline Quantil 10 & $1.640,40$ & $2.226,30$ & $1.971,80$ & $2.567,10$ & $1.635,90$ & $2.704,30$ \\
\hline Quantil 50 & $7.792,10$ & $11.917,40$ & $6.721,00$ & $9.027,70$ & $5.891,60$ & $8.384,30$ \\
\hline Quantil 90 & $22.077,70$ & $28.571,20$ & $18.160,30$ & $26.041,60$ & $18.259,20$ & $25.618,70$ \\
\hline $\mathrm{RQ}(90-10)$ & $20.437,30$ & $26.344,90$ & $16.188,50$ & $23.474,50$ & $16.623,30$ & $22.914,40$ \\
\hline $\mathrm{RQ}(90-50)$ & $14.285,60$ & $16.653,80$ & $11.439,30$ & $17.013,90$ & $12.367,60$ & $17.234,40$ \\
\hline $\mathrm{RQ}(50-10)$ & $6.151,70$ & $9.691,10$ & $4.749,20$ & $6.460,60$ & $4.255,70$ & $5.680,00$ \\
\hline
\end{tabular}

Nota: individuos entre 18 y 65 años con ingresos laborales positivos. El nivel educativo básica primaria incluye los individuos con preescolar y primaria (grado $1 .^{\circ}$-grado $5 .^{\circ}$ ), el nivel educativo media incluye los individuos con secundaria (grado $6 .^{\circ}$ - grado 9..$^{\circ}$ y educación media (grado $10 .^{\circ}$ - gradol1..$^{\circ}$, y el nivel educativo contiene los individuos con educación universitaria o superior.

Fuente: elaboración propia con base en las encuestas del programa Mesep-DANE. 


\section{RESULTADOS}

La tabla 2 muestra la descomposición Oaxaca (1973) y Blinder (1973) del salario horario promedio por género en Colombia durante el periodo de estudio, sin corrección y con corrección por sesgo de selección. Como se puede observar, la brecha bruta del salario laboral horario promedio disminuye en el tiempo. Para el año 2004 esta fue de $14 \%$, con una variación promedio negativa de once puntos porcentuales, lo que conlleva a una brecha para el año 2012 de 11 \%. Por su parte, el efecto característica, decrece con el paso de los años, al ser positivo para el año 2004 (1,6 \%), puesto que para los años 2008 y 2012 fue de -12 \% y -17 \% respectivamente. Este resultado determina que, en promedio, la diferencia en las características productivas es a favor de las mujeres, es decir, los componentes que mejoran la productividad como es el caso de la educación es mayor en las mujeres con relación a los hombres.

Por su parte, el efecto remuneración, que indica cuál sería el diferencial salarial de las mujeres si fueran remuneradas como los hombres, es positivo y creciente durante el periodo de estudio (13\% para 2004, $24 \%$ para 2008 y $29 \%$ para 2012), mayor que la brecha total del ingreso laboral horario, lo cual hace que el efecto característica sea compensado, lo que genera un diferencial salarial en favor de los hombres.

Tabla 2. Brecha bruta. Descomposición de Oaxaca (1973)-Blinder (1973).

\begin{tabular}{|l|c|c|c|c|c|c|}
\cline { 2 - 7 } \multicolumn{1}{c|}{} & \multicolumn{3}{c|}{ Sin corregir por sesgo de selección } & \multicolumn{3}{c|}{ Corrección por sesgo de selección } \\
\cline { 2 - 7 } \multicolumn{1}{c|}{ Brecha bruta } & 2004 & 2008 & 2012 & 2004 & 2008 & 2012 \\
\hline \multirow{2}{*}{ Efecto características } & $0,14^{* * *}$ & $0,12^{* * *}$ & $0,11^{* * *}$ & $0,14^{* * *}$ & $0,12^{* * *}$ & $0,11^{* * *}$ \\
\cline { 2 - 7 } & $-0,0033$ & $-0,03$ & $-0,002$ & $-0,0033$ & $-0,0026$ & $-0,0018$ \\
\cline { 2 - 7 } & $0,016^{* * *}$ & $-0,12^{* * *}$ & $-0,17^{* * *}$ & $-0,051^{* * *}$ & $-0,23^{* * *}$ & $-0,28^{* * *}$ \\
\hline \multirow{2}{*}{ Efecto remuneración } & $-0,0032$ & $-0,02$ & $-0,001$ & $-0,0036$ & $-0,0033$ & $-0,0029$ \\
\cline { 2 - 7 } & $0,13^{* * *}$ & $0,24^{* * *}$ & $0,29^{* * *}$ & $0,20^{* * *}$ & $0,35^{* * *}$ & $0,40^{* * *}$ \\
\hline & $-0,0031$ & $-0,02$ & $-0,014$ & $-0,0036$ & $-0,0033$ & $-0,0029$ \\
\hline
\end{tabular}

Nota: ${ }^{*} \mathrm{p}<0,1 ;{ }^{* *} \mathrm{p}<0,05 ;{ }^{* * *} \mathrm{p}<0,01$. Individuos entre 18 y 65 años con ingresos laborales positivos.

Fuente: elaboración propia con base en las encuestas del programa Mesep-DANE.

Al controlar por sesgo de selección, las estimaciones obtenidas muestran que el efecto característica en este caso es negativo y decreciente para todos los años analizados (es decir - 5 \% en 2004, -23\% en 2008 y - $28 \%$ en 2012). Por su parte, el efecto remuneración es creciente. Sin embargo, este es mayor con relación al modelo de Oaxaca-Blinder sin corregir por sesgo de selección (20 \% para 2004, 35 \% para 2008 y $40 \%$ para 2012). Estos resultados reflejan que existe una alta discriminación en contra de las mujeres y que esta se incrementa, al incluir como instrumento una variable que influya en el tiempo de trabajo de las mujeres, como es el caso de los hijos menores. 
La tabla 3 muestra los resultados de la descomposición de la brecha salarial para los diversos cuantiles de la distribución del salario horario. La columna 1 presenta la brecha bruta, efecto característica y efecto remuneración, al controlar por características sociodemográficas y ocupacionales; la columna 2 incluye las variables regionales. Por su parte, cada fila determina los distintos cuantiles (q10, q25, q50, q75, q90) de la distribución del salario horario.

Como se puede observar, existe un comportamiento creciente de la brecha bruta en la distribución del salario para cada año de estudio, es decir, a medida que los hombres se ubican en ingresos laborales más altos, el diferencial salarial con respecto a las mujeres se incrementa. Al controlar por características sociodemográficas (educación y edad) y ocupacionales (trabajador privado, del gobierno, doméstico y cuenta propia), el diferencial salarial por género es mayor en los cuantiles más altos de la distribución del ingreso laboral correspondiente a cada uno de los años utilizados, es decir, se evidencia el fenómeno de techo de cristal en la población femenina.

Tabla 3. Brecha bruta, efecto característica y efecto remuneración. Metodología Melly (2005).

\begin{tabular}{|c|c|c|c|c|c|c|c|}
\hline & \multicolumn{2}{|c|}{ Brecha bruta } & \multicolumn{2}{|c|}{ Efecto característica } & \multicolumn{2}{|c|}{ Efecto remuneración } \\
\hline & & (1) & (2) & (1) & (2) & (1) & (2) \\
\hline \multirow{5}{*}{2004} & $\mathrm{q} 10$ & $0,26^{* * *}$ & $0,25^{* * *}$ & $0,06^{* * *}$ & $0,07^{* * *}$ & $0,20^{* * *}$ & $0,18^{* * *}$ \\
\hline & $\mathrm{q} 25$ & $0,27^{* * *}$ & $0,26^{* * *}$ & $0,10^{* * *}$ & $0,10^{* * *}$ & $0,16^{* * *}$ & $0,16^{* * *}$ \\
\hline & $\mathrm{q} 50$ & $0,32^{* * *}$ & $0,31^{* * *}$ & $0,13^{* * *}$ & $0,14^{* * *}$ & $0,19^{* * *}$ & $0,17^{* * *}$ \\
\hline & $\mathrm{q} 75$ & $0,39^{* * *}$ & $0,40^{* * *}$ & $0,18^{* * *}$ & $0,18^{* * *}$ & $0,21^{* * *}$ & $0,22^{* * *}$ \\
\hline & q90 & $0,45^{* * *}$ & $0,46^{* * *}$ & $0,20^{* * *}$ & $0,20^{* * *}$ & $0,25^{* * *}$ & $0,26^{* * *}$ \\
\hline \multirow{5}{*}{2008} & $\mathrm{q} 10$ & $0,31^{* * *}$ & $0,31^{* * *}$ & $0,02^{* * *}$ & $0,02^{* * *}$ & $0,29^{* * *}$ & $0,29^{* * *}$ \\
\hline & $\mathrm{q} 25$ & $0,34^{* * *}$ & $0,34^{* * *}$ & $0,05^{* * *}$ & $0,05^{* * *}$ & $0,29^{* * *}$ & $0,29^{* * *}$ \\
\hline & $\mathrm{q} 50$ & $0,41^{* * *}$ & $0,41^{* * *}$ & $0,13^{* * *}$ & $0,12^{* * *}$ & $0,28^{* * *}$ & $0,29^{* * *}$ \\
\hline & q75 & $0,46^{* * *}$ & $0,46^{* * *}$ & $0,19^{* * *}$ & $0,19^{* * *}$ & $0,27^{* * *}$ & $0,27^{* * *}$ \\
\hline & q90 & $0,47^{* * *}$ & $0,47^{* * *}$ & $0,21^{* * *}$ & $0,20^{* * *}$ & $0,26^{* * *}$ & $0,27^{* * *}$ \\
\hline \multirow{5}{*}{2012} & $\mathrm{q} 10$ & $0,31^{* * *}$ & $0,31^{* * *}$ & $-0,09^{* * *}$ & $-0,09^{* * *}$ & $0,40^{* * *}$ & $0,40^{* * *}$ \\
\hline & q25 & $0,28^{* * *}$ & $0,28^{* * *}$ & $-0,06^{* * *}$ & $-0,07^{* * *}$ & $0,34^{* * *}$ & $0,35^{* * *}$ \\
\hline & $\mathrm{q} 50$ & $0,30^{* * *}$ & $0,31^{* * *}$ & $-0,02^{* * *}$ & $-0,02^{* * *}$ & $0,32^{* * *}$ & $0,33^{* * *}$ \\
\hline & $\mathrm{q} 75$ & $0,37^{* * *}$ & $0,37^{* * *}$ & $0,05^{* * *}$ & $0,05^{* * *}$ & $0,32^{* * *}$ & $0,32^{* * *}$ \\
\hline & q90 & $0,42^{* * *}$ & $0,42^{* * *}$ & $0,12^{* * *}$ & $0,12^{* * *}$ & $0,30^{* * *}$ & $0,30^{* * *}$ \\
\hline \multicolumn{8}{|c|}{ Controles: } \\
\hline \multicolumn{2}{|c|}{ Educación, edad y ocupación } & Sí & Sí & Sí & Sí & Sí & Sí \\
\hline \multicolumn{2}{|c|}{ Región } & No & Sí & No & Sí & No & Sí \\
\hline
\end{tabular}

Nota: ${ }^{*} \mathrm{p}<0,1 ;{ }^{* *} \mathrm{p}<0,05 ;{ }^{* * *} \mathrm{p}<0,01$. Individuos entre 18 y 65 años con ingresos laborales positivos.

Fuente: elaboración propia con base en las encuestas del programa Mesep-DANE. 
No obstante, si bien es cierto que la brecha salarial es creciente para los diferentes cuantiles de la distribución de salario horario, con el paso del tiempo se ha reducido con alguna variabilidad en los diferentes segmentos de la distribución. La brecha bruta de los individuos, en la cola inferior de la distribución (cuantil 10), durante el año 2004 es de $25 \%$, en 2008 y 2012 fue $31 \%$. Con respecto a los individuos con mayores ingresos laborales (cuantil 90), los resultados varían para el año 2004, la brecha observada es de $45 \%$, en 2008 fue $47 \%$ y para el 2012 estuvo en torno al $42 \%$, este comportamiento se debe a los cambios en los efectos característica y remuneración.

El efecto característica ha tenido un comportamiento decreciente para los individuos con bajos (cuantil 10) y mayores (cuantil 90) ingresos en la distribución de salarios. Para el año 2004 este fue de 7 \% y $20 \%$, en 2008 fue 2 \% y $20 \%$ y para 2012 fue $-9 \%$ y $12 \%$, en otras palabras, para los intervalos con menores ingresos, la diferencia entre las características productivas (educación) entre hombres y mujeres es menor con respecto a la cola superior de la distribución de los salarios. El efecto remuneración es importante, debido a que explica el diferencial salarial en cada uno de los percentiles de la distribución del salario horario, ya que este efecto incrementa la brecha bruta. Lo primero que se puede determinar es el comportamiento creciente del efecto remuneración para el año 2004 con un 18 \% para los individuos con menores salarios (cuantil 10) y $25 \%$ para los individuos con mayores salarios (cuantil 90). En 2008 fue $29 \%$ y $27 \%$ para los individuos con menores y mayores ingresos, para 2012 estuvo alrededor de $40 \%$ para los individuos con salarios menores (cuantil 10) y $30 \%$ para los individuos con elevados salarios (cuantil 90).

La tabla 4 muestra la brecha bruta, el efecto característica y el efecto remuneración para los diferentes cuantiles de la distribución del salario horario al corregir por sesgo de selección. Como se puede observar, al Incluir como instrumento la tenencia de hijos en el hogar, el efecto característica cambia para toda la distribución de salarios. Las estimaciones muestran que los individuos en la cola izquierda (cuantil 10) y derecha (cuantil 90) de la distribución de salarios, tienen un efecto de 5 \% y $15 \%$ para el año 2004, -6 \% y 8 \% en 2008 y - 15 \% y $3 \%$ para 2012. Si bien este efecto sigue creciente al pasar por los diferentes cuantiles del salario horario, al ser comparado con los resultados obtenidos (tabla 3), se evidencia una sobreestimación en el efecto característica al no haber controlado por sesgo de selección, una diferencia para los individuos con menores (cuantil 10) y mayores (cuantil 90) ingresos de 2 \% y 5 \% en 2004, de $8 \%$ y 12 \% en 2008, 6 \% y $9 \%$ para el año 2012.

El efecto remuneración es creciente durante el periodo de estudio para cada uno de los percentiles de la distribución del salario horario. Las estimaciones 
muestran que los individuos con menor salario horario (cuantil 10) para el año 2004 tienen un efecto de 20 \%, en 2008 es de 37 \% y 2012 llega al 46 \%. Con respecto al $10 \%$ de los hombres y mujeres con mayor salario (cuantil 90), el efecto remuneración para el 2004 es de 31 \%, para el 2008 y en 2012 alcanza una cifra de 39 \%. Los resultados obtenidos determinan que la brecha salarial explicada por las diferencias en las remuneraciones de capital humano (incluida la discriminación salarial) de los hombres con relación a las mujeres disminuye si se tienen hijos menores en los hogares, analizado como el sobrecosto en el salario de eficiencia de las mujeres.

Tabla 4. Brecha bruta, efecto características y efecto remuneración. Metodología Melly (2005) y Buchinsky (1998) con corrección por sesgo de selección.

\begin{tabular}{|c|c|c|c|c|c|c|c|}
\hline & \multicolumn{2}{|c|}{ Brecha bruta } & \multicolumn{2}{|c|}{ Efecto características } & \multicolumn{2}{|c|}{ Efecto remuneración } \\
\hline & & (1) & (2) & (1) & (2) & (1) & (2) \\
\hline \multirow{5}{*}{2004} & $\mathrm{q} 10$ & $0,26^{* * *}$ & $0,25^{* * *}$ & $0,12^{* * *}$ & $0,05^{* * *}$ & $0,14^{* * *}$ & $0,20^{* * * *}$ \\
\hline & $\mathrm{q} 25$ & $0,27^{* * *}$ & $0,26^{* * *}$ & $0,14^{* * *}$ & $0,08^{* * *}$ & $0,13^{* * *}$ & $0,18^{* * *}$ \\
\hline & $q 50$ & $0,32^{* * *}$ & $0,31^{* * *}$ & $0,16^{* * *}$ & $0,10^{* * *}$ & $0,16^{* * *}$ & $0,21^{* * *}$ \\
\hline & q75 & $0,39^{* * *}$ & $0,40^{* * *}$ & $0,18^{* * *}$ & $0,14^{* * *}$ & $0,21^{* * *}$ & $0,26^{* * *}$ \\
\hline & q90 & $0,45^{* * *}$ & $0,46^{* * *}$ & $0,19^{* * *}$ & $0,15^{* * *}$ & $0,26^{* * *}$ & $0,31^{* * *}$ \\
\hline \multirow{5}{*}{2008} & $\mathrm{q} 10$ & $0,31^{* * *}$ & $0,31^{* *}$ & $-0,04^{* * *}$ & $-0,06^{* * *}$ & $0,35^{* * *}$ & $0,37^{* * *}$ \\
\hline & q25 & $0,34^{* * *}$ & $0,34^{* * *}$ & $0,01^{* * *}$ & $-0,02^{* * *}$ & $0,31^{* * *}$ & $0,36^{* * *}$ \\
\hline & q50 & $0,41^{* * *}$ & $0,41^{* * *}$ & $0,09^{* * *}$ & $0,04^{* * *}$ & $0,32^{* * *}$ & $0,37^{* * *}$ \\
\hline & q75 & $0,46^{* * *}$ & $0,46^{* * *}$ & $0,14^{* * *}$ & $0,09^{* * *}$ & $0,32^{* * *}$ & $0,37^{* * *}$ \\
\hline & q90 & $0,47^{* * *}$ & $0,47^{* * *}$ & $0,15^{* * *}$ & $0,08^{* * *}$ & $0,32^{* * *}$ & $0,39^{* * *}$ \\
\hline \multirow{5}{*}{2012} & $\mathrm{q} 10$ & $0,31^{* * *}$ & $0,31^{* * *}$ & $-0,06^{* * *}$ & $-0,15^{* * *}$ & $0,37^{* * *}$ & $0,46^{* * *}$ \\
\hline & q25 & $0,28^{* * *}$ & $0,28^{* * *}$ & $-0,04^{* * *}$ & $-0,13^{* * *}$ & $0,32^{* * *}$ & $0,41^{\cdots *}$ \\
\hline & $q 50$ & $0,30^{* * *}$ & $0,31^{* * *}$ & $-0,01^{* * *}$ & $-0,09^{* * *}$ & $0,31^{* * *}$ & $0,40^{* * *}$ \\
\hline & $\mathrm{q} 75$ & $0,37^{* * *}$ & $0,37^{* * *}$ & $0,06^{* * *}$ & $-0,03^{* * *}$ & $0,31^{* * *}$ & $0,40^{* * * *}$ \\
\hline & q90 & $0,42^{* * *}$ & $0,42^{* * *}$ & $0,12^{* * *}$ & $0,03^{* * *}$ & $0,30^{* * *}$ & $0,39^{* * *}$ \\
\hline \multicolumn{8}{|c|}{ Controles: } \\
\hline \multicolumn{2}{|c|}{ Educación, edad y ocupación } & Sí & Sí & Sí & Sí & Sí & Sí \\
\hline \multicolumn{2}{|c|}{ Región } & No & Sí & No & Sí & No & Sí \\
\hline
\end{tabular}

Nota: ${ }^{*} p<0,1 ;{ }^{* *} p<0,05 ;{ }^{* *} p<0,01$. Individuos entre 18 y 65 años con ingresos laborales positivos.

Fuente: elaboración propia con base en las encuestas del programa Mesep-DANE.

\section{CONCLUSIONES}

Este estudio presenta evidencia sobre el diferencial salarial por género en Colombia durante el periodo 2004-2012. Los resultados obtenidos muestran que la brecha 
bruta ha disminuido con el paso del tiempo con una variación promedio negativa de 11 puntos porcentuales. Como era de esperarse, de acuerdo a la literatura de diferencial salarial por género, el efecto remuneración (componentes no explicados), explica una parte mayor de la brecha bruta, la cual es creciente a medida que pasa el tiempo. Por su parte, el efecto característica, tiene un peso cada vez menor en explicar el diferencial salarial por género en Colombia, ya que, con el paso de los años, este se hace negativo, lo que indica que en promedio la diferencia en las características productivas es a favor de las mujeres.

De igual manera, al utilizar como variable de selección la tenencia de hijos menores de 18 años en el hogar, con el propósito de estimar la inversa de Mills y corregir por sesgo de selección. Las estimaciones muestran que la brecha bruta promedio no cambia, sigue en favor de los hombres, aunque decrece con el paso del tiempo. El efecto característica del salario promedio es negativo y decreciente para todos los años analizados. Por su parte, el efecto remuneración continúa positivo y creciente, aunque este es mayor con relación a las estimaciones sin corregir por sesgo de selección. Estos resultados reflejan que existe una alta discriminación en contra de las mujeres y que esta se incrementa al adicionar como instrumento una variable que intervenga en el tiempo de trabajo de las mujeres.

Al analizar la brecha bruta para toda la distribución de los salarios, se encuentra que esta es creciente para los diferentes niveles de ingresos laborales en cada año de estudio, es decir, el diferencial salarial por género es mayor en los cuantiles más altos de la distribución del ingreso laboral. Esto valida la hipótesis de la existencia de techos de cristal para la población femenina con respecto al ingreso laboral. Así mismo, al controlar por las características productivas y regionales, se obtiene que el efecto característica ha tenido un comportamiento decreciente durante el periodo analizado para los individuos con menores (cuantil 10) y mayores (cuantil 90) ingresos en la distribución de salarios, esto es, la diferencia en las características productivas, para los intervalos con menores ingresos entre hombres y mujeres, es menor con respecto a la cola superior de la distribución de los salarios.

Por otro lado, el efecto remuneración es el componente que más explica el diferencial salarial en los diferentes puntos de la distribución del salario horario, ya que el efecto remuneración incrementa la brecha bruta al compensar el valor negativo del efecto característica.

En cuanto a la corrección por sesgo de selección en la distribución del ingreso laboral, se encuentra que el efecto característica, presenta un comportamiento decreciente con el paso del tiempo. Sin embargo, este efecto es menor con relación al efecto característica, que no incluye la corrección por sesgo de selección. En otras 
palabras, las mujeres con menores ingresos (cuantil 10), tienen mejores características productivas que los hombres (efecto característica negativo). En cambio, en la cola derecha de la distribución del salario (cuantil 90), son los hombres los que poseen mayores características de capital humano. Con respecto al efecto remuneración, se determina que es creciente para cada uno de los años analizados; no obstante, al compararse con el efecto remuneración, sin corregir por sesgo de selección, se encuentra una subestimación, es decir, las diferencias en las remuneraciones de capital humano entre hombre y mujeres aumentan al corregir por sesgo de selección, al ser los individuos con menores ingresos (cuantil 10) los que presenta mayor diferencia.

Estos resultados sugieren que una política pública en educación e inclusión laboral (incluye educación infantil) sería adecuada para que las mujeres puedan dejar de dedicarse a actividades del hogar (rol de madre) y puedan acceder al mercado laboral. Dado esto, el gobierno nacional creó y aprobó la Ley 1496 de 2011, la cual tiene como objetivo garantizar la igualdad salarial entre hombres y mujeres, al fijar los mecanismos necesarios para que dicha igualdad sea real. Es por ello que, sus diez artículos plantean definir los conceptos de discriminación laboral, crear los factores de valoración salarial, realizar el seguimiento para verificar los avances en materia de igualdad laboral y por último medir los avances con ayuda de las encuestas a los hogares. Estás políticas pueden ser útiles a la hora de combatir la discriminación laboral por género en sociedades donde existen restricciones en el acceso a la educación y a oportunidades laborales.

Para finalizar, se debe establecer que siempre existirán problemas en la estimación en estos modelos, ya que existen sesgos por variables omitidas no observables. Estos problemas afectan a la distribución del salario, lo cual determina que el efecto remuneración no se explique por la discriminación salarial. No obstante, poder controlar por sesgo de selección es un avance para mejorar el análisis de esta problemática del mercado laboral.

\section{BIBLIOGRAFÍA}

Abadía Alvarado, Luz Karime (2005). Discriminación salarial por sexo en Colombia: un análisis desde la discriminación estadística. Bogotá, Colombia: Pontificia Universidad Javeriana, 63 p.

Arrow, Kenneth (1971). The Theory of Discrimination. Industrial Relations Section Working Paper. Nueva Jersey, Estados Unidos de América: Princeton University, 35 p.

Autor, David; Katz, Lawrence y Kearney, Melissa (2005). Rising Wage Inequality: The Role of Composition and Prices. En: NBER Working Paper, n. ${ }^{\circ}$ 11628, p. 1-66.

Becker, Gary Stanley (1971). The Economics of Discrimination. Chicago, Estados Unidos de América: University of Chicago Press 167 p. 
Bernat, Luisa; Ribero, Rocío y Tenjo, Jaime (2005). Evolución de las diferencias salariales por sexo en seis países de América Latina: un intento de interpretación. En: Documento CEDE, n. ${ }^{\circ} 18$, p. $1-59$.

Blinder, Alan (1973). Wage Discrimination: Reduced Form and Structural Estimates. En: Journal of Human Resources, vol. 8, n. ${ }^{\circ}$, otoño, p. 436-455.

Buchinsky, Moshe (1998). Recent Advances in Quantile Regression Models: A Practical Guideline for Empirical Research. En: The Journal of Human Resources, vol. 33, n. ${ }^{\circ}$ 1, invierno, p. 88-126.

DANE $\square$ Departamento Administrativo Nacional de Estadística $\square$ (2012). Metodología Gran Encuesta Integrada de Hogares. Bogotá, Colombia: DANE, 1239 p.

Fernández, María del Pilar (2006). Determinantes del diferencial salarial por género en Colombia 1997-2003. En: Documento CEDE, n. ${ }^{\circ}$ 2006-32, Universidad de Los Andes, p. 165-208.

Flórez, Carmen (2004). la transformación de los hogares: una visión de largo plazo. En: Revista Coyuntura Social, n. ${ }^{\circ}$ 30, junio, p. 23-49.

Firpo, Sergio; Fortin, Nicole y Lemieux, Thomas (2011). Decomposition Methods in Economics. En: NBER Working Paper, n. ${ }^{\circ} 16045$, p. 1-118.

Goldin, Claudia y Rouse, Cecilia (2000). Orchestrating Impartiality: The Impact of Blind Auditions on Female Musicians. En: American Economic Review, vol. 90, n. ${ }^{\circ}$ 4, septiembre, p. 715-741.

Galvis, Luis Armando (2010). Diferenciales salariales por género y región en Colombia: una aproximación con regresión por cuantiles. En: Revista de Economía del Rosario, vol. 13, n. ${ }^{\circ}$, diciembre, p. 235-277.

Heckman, James (1979). Sample Selection Bias as a Specification Error. En: Econometrica, vol. 47, n. ${ }^{\circ}$, enero, p. 153-161.

Koenker, Roger y Bassett, Gilbert. (1978). Regression Quantiles. En: Econométrica, vol. 46, n. ${ }^{\circ}$ 1, p. 33-50.

Machado, José y Mata, José (2005). Counterfactual Decomposition of Changes in Wage Distributions Using Quantile Regression. En: Journal of Applied Econometrics, vol. 20, n. ${ }^{\circ}$ 4, p. 445-465.

Melly, Blaise (2005). Decomposition of Differences in Distributions Using Quantile Regression. En: Labour Economics, vol. 12, n. ${ }^{\circ}$, agosto, p. 577-590.

Oaxaca, Ronald (1973). Male - Female Wage Differentials in Urban Labor Markets. En: International Economics Review, vol. 14, n. 3, octubre, p. 693-709.

Phelps, Edmund (1972). The Statistical Theory of Racism and Sexism. En: American Economic Review, vol. 62, n. 4, septiembre, p. 659-661.

Ribero, Rocío y Meza, Claudia. (1997). Los ingresos laborales de hombres y mujeres en Colombia: 1976 1995. En: Archivos de Macroeconomía, n. 63 , p.1-31.

Tenjo, Jaime (1993). 1976-1989: cambios en los diferenciales salariales entre hombres y mujeres. En: Planeación y Desarrollo, vol. 24, p. 103-116.

Urdinola Contreras, Piedad (1998). Mujeres en sus casas: un recuento de la población femenina económicamente activa. En: Archivos de Macroeconomía, n. ${ }^{\circ}$ 85, p.1-31. 


\section{ANEXOS}

Figura A1. Funciones de densidad del logaritmo natural del salario horario para hombres y mujeres.
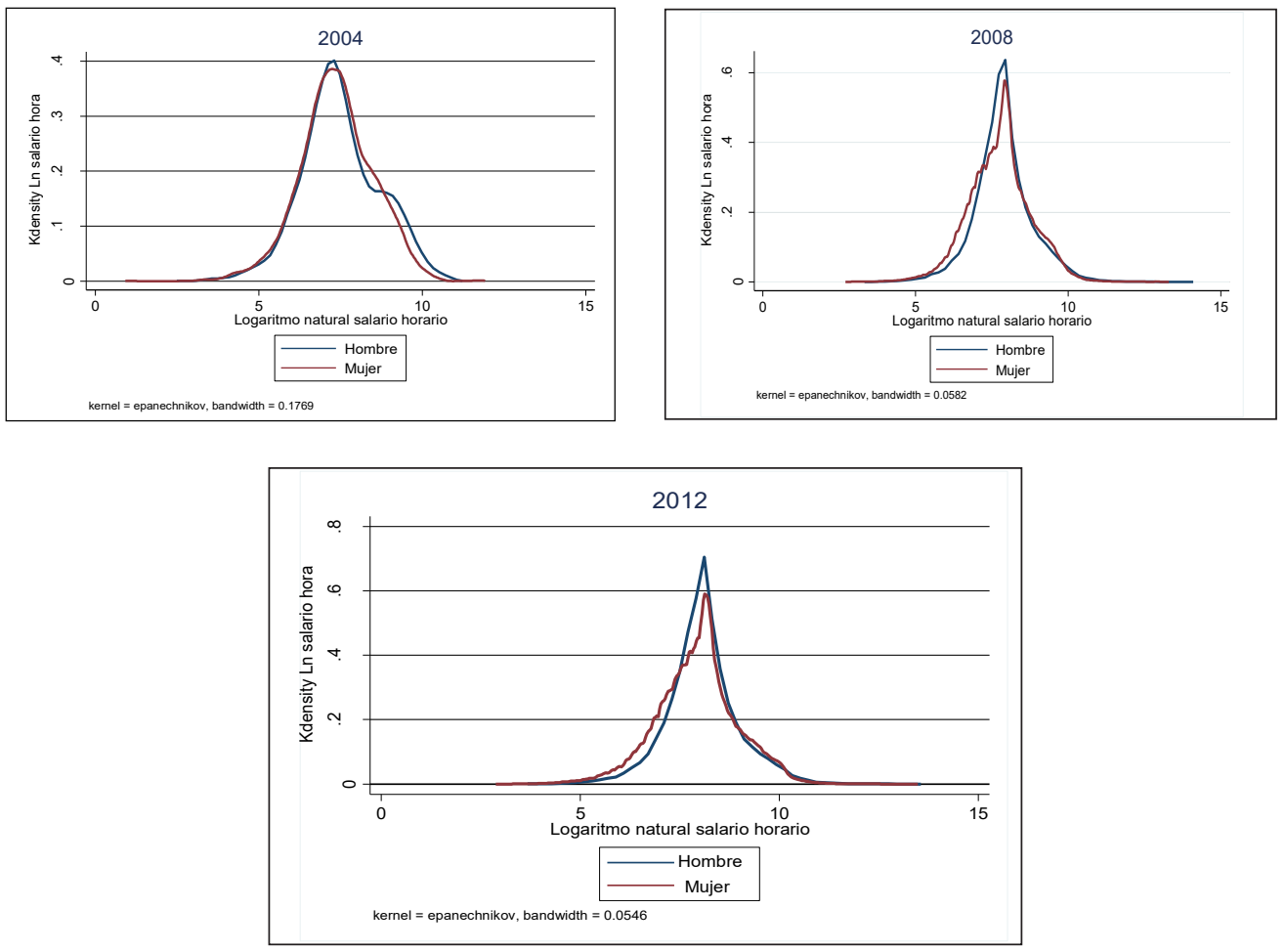

Nota: individuos entre 18 y 65 años con ingresos laborales positivos.

Fuente: elaboración propia con base en las encuestas del programa Mesep-DANE. 
Figura A2. Brecha bruta, efecto características y efecto remuneración por cuantiles de ingreso, al controlar por características sociodemográficas, ocupacionales y regionales.
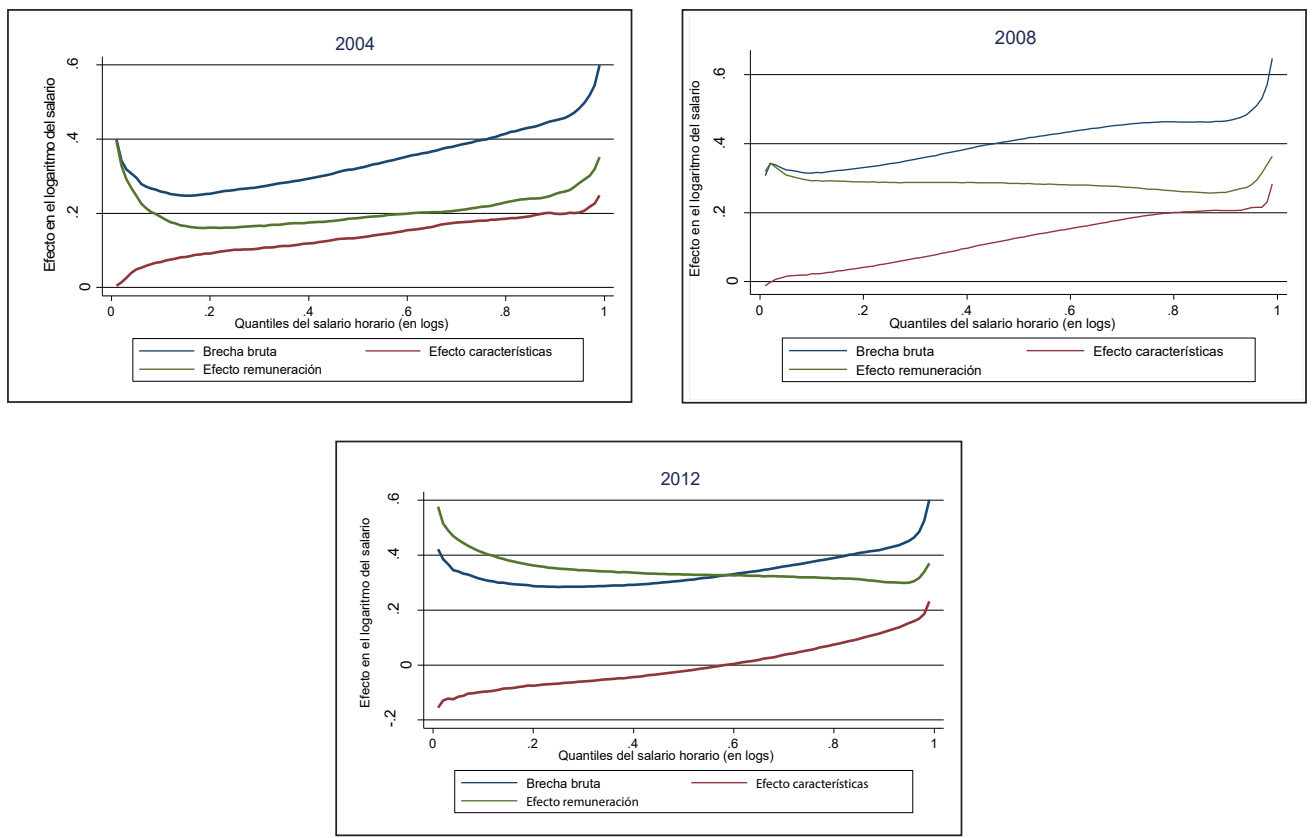

Nota: individuos entre 18 y 65 años con ingresos laborales positivos.

Fuente: elaboración propia con base en las encuestas del programa Mesep-DANE. 
Figura A3. Brecha bruta, efecto características y efecto remuneración por cuantiles de ingreso, al controlar por características sociodemográficas, ocupacionales y regionales, con corrección por sesgo de selección.
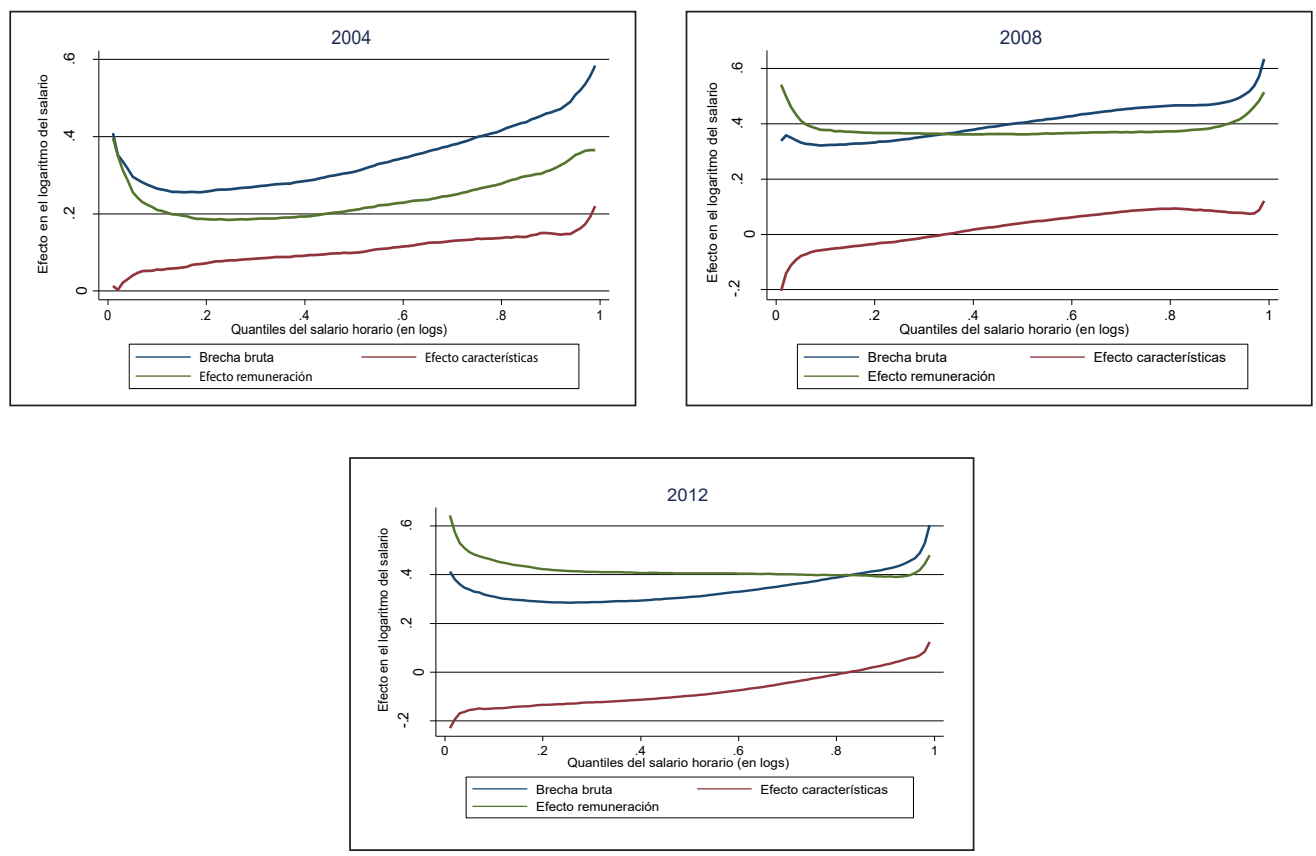

Nota: Individuos entre 18 y 65 años con ingresos laborales positivos.

Fuente: elaboración propia con base en las encuestas del programa Mesep-DANE. 